\section{The effect of in vitro exposure to antisense oligonucleotides on macrophage morphology and function}

\author{
Ann Brasey, ${ }^{1}$ Raouf Igue, ${ }^{1}$ \\ Loubna Djemame, ${ }^{1}$ Serge Séguin, ${ }^{1}$ \\ Paolo Renzi, ${ }^{2}$ Nicolay Ferrari, ${ }^{1}$ \\ Rosanne Séguin ${ }^{1}$ \\ ${ }^{1}$ Topigen Pharmaceuticals Inc., part of \\ Pharmaxis group; ${ }^{2} \mathrm{CHUM}$ Research \\ Center, Notre-Dame Hospital, Montréal, \\ Québec, Canada
}

\section{Abstract}

Antisense oligonucleotides (AON) delivered via inhalation are in drug development for respiratory diseases. In rodents and monkeys, repeated exposure to high doses of inhaled phosphorothioate (PS) AON can lead to microscopic changes in the lungs, including accumulation of alveolar macrophages in the lower airway that have a foamy appearance. The functional consequences that result from this morphological change are unclear as there is controversy whether the vacuoles/inclusion bodies reflect normal clearance of the inhaled AON or are early indicators of lung toxicity. The morphological and functional responses of macrophage to PS AON were characterized in vitro using the comparator drug amiodarone, as a known inducer of foamy macrophages. Morphological changes of increased vacuolization with the presence of lamellated structures were observed in macrophages in response to both amiodarone and AON treatment. Functional responses to the drugs clearly differed with amiodarone treatment leading to apoptosis of cells and cell death, release of proinflammatory mediators IL-1RA, MIP- $1 \alpha$ and TNF $\alpha$, decrease in IP-10, a cytokine shown to be involved in protection against pulmonary fibrosis and altered phagocytosis capacity of the cells. In contrast, $\mathrm{AON}$ in concentrations up to $30 \mu \mathrm{M}$, had no effect on cell viability or apoptosis, had minimal effects on pro-inflammatory cytokines, increased IP-10 levels and did not alter the phagocytic capacity of the cells. Exposure of macrophages to AON in vitro, led to morphological changes of increased vacuolization, but did not lead to functional consequences which were observed with another vacuolization-inducing drug, suggesting that the in vivo phenotypic changes observed following inhalation of AON may be consistent with a clearance mechanism and not an activation or impairment of macrophages.

\section{Introduction}

Oligonucleotides (ONs) are currently being advanced as potential new drug therapies for a wide array of indications, including respiratory diseases. Local administration to the lung can be approached non-invasively by oral inhalation, using aerosolized ONs. Pulmonary bioavailability with concurrent low systemic levels has been reported using areosolized antisense oligonucleotides (AONs), ${ }^{1-2}$ presenting the opportunity of treating local respiratory diseases with direct pulmonary doses and minimizing the exposure of non-target tissues. In addition, the large absorption surface area of the lung ${ }^{1}$ and its lining with cationic lipid-containing surfactant has been proposed to facilitate AON uptake within the lungs. ${ }^{3}$ The efficacy of AONs delivered directly to the lungs to reduce inflammatory diseases has been demonstrated for a number of targets in rodents ${ }^{3-4}$ and monkeys. ${ }^{5}$ Furthermore, the utility of this modality has also been established in clinical studies. ${ }^{6}$

The toxicity of ONs has been well characterized and appears mainly related to the chemical class of the $0 \mathrm{~N},{ }^{7}$ rather than being related to the mechanism of action (e.g., antisense activity). For some types of ONs particularly those containing the phosphorothioate (PS) backbone modification, local inflammation at the site of injection is commonly observed (e.g., with subcutaneous, intravenous, intraperitoneal, and intraocular routes) ${ }^{8}$ This response typically does not present an issue for clinical safety, particularly when the injection site can be changed with each injection (e.g., with intravenous or subcutaneous injection). However for some routes, such as intraocular and pulmonary, a local inflammatory response may be more nocuous. To date, there are only a few reports describing the toxicity of PS ONs administered by inhalation. It has been reported that repeated pulmonary administration of a PS AON on a subacute basis (e.g., 2 weeks) can lead to microscopic changes in the respiratory tract of rodents and monkeys. ${ }^{2,9}$ The most common alterations observed can be categorized into two types of responses: i) a mononuclear cell infiltrate located within the pulmonary interstitium and other parenchymal regions; and ii) an accumulation of macrophages in the alveolar region [i.e., an increase in the number of alveolar macrophages (AMs)]. The former response (mononuclear cell infiltration) may be akin to the inflammation at sites of injection that is observed when ONs are given by other routes (as discussed above), ${ }^{8}$ whereas the influx of AMs has been speculated to be non-inflammatory in nature and possibly more of a non-specific response to an inhaled foreign material,
Correspondence: Rosanne Seguin, Topigen Pharmaceuticals Inc., 2901 Rachel East, Suite 13, Montreal QC, H1W 4A4, Canada.

Tel. +1.514.868.0077 - Fax: +1.514.868-0011.

E-mail rosanne.seguin@topigen.com

Key words: antisense, macrophage, morphology, phagocytosis, phospholipid accumulation.

Acknowledgements: Dr. Stephen Groom of CanBioPharma Consulting, Inc., characterized the morphology of the Hema-3-stained RAW 264.7 cells and cytospins of BAL. Jeannie Mui and Dr. Hojatollah Vali of the Department of Anatomy and Cell Biology at McGill University obtained the electron microscopic photographs. Dr.Doug Kornbrust reviewed the manuscript.

Contributions: AB, RI, LD, SS, experimental research, cell manipulations and data analyses $\mathrm{AB}, \mathrm{PR}, \mathrm{NF}, \mathrm{RS}$, study design, data analyses and writing of manuscript.

Funding: AB, RI, LD, SS, NF and RS are/were employed by Topigen at the time of the research. PR is a consultant for Topigen/ Pharmaxis.

Conflict of interest: the authors report no conflicts of interest.

Received for publication: 13 September 2011. Revision received: 12 October 2011.

Accepted for publication: 17 October 2011.

This work is licensed under a Creative Commons Attribution NonCommercial 3.0 License (CC BYNC 3.0).

(C) Copyright A. Brasey et al., 2011

Licensee PAGEPress, Italy

Journal of Nucleic Acids Investigation 2011; 2:e12 doi:10.4081/jnai.2011.e12

as such accumulation of AMs is often observed with inhalation of other substances. ${ }^{10-11}$ In some cases, the AMs appear to be vacuolated (sometimes described as foamy), suggesting that they may contain inhaled $\mathrm{ON}$, which is consistent with the view that this response may be part of a natural clearance mechanism mediated by the resident macrophages in the lungs. However, the nature of this AM response to inhaled PS ONs has not been elucidated.

The accumulation of foamy AMs has also been reported to occur following oral administration of some drugs, such as the antiarryhthmic drug amiodarone. ${ }^{12-15}$ Approximately 5\% of amiodarone-treated patients have adverse lung effects. ${ }^{16}$ Although the bronchoalveolar lavage (BAL) of patients exposed to amiodarone exhibit a characteristic finding of morphologic abnormalities in BAL macrophage, specifically vacuolation rendering them foamy ${ }^{16}$ neither the number nor appearance of foamy macrophages is considered to be a predictor of amiodarone-tox- 
icity, as they can be observed even in non-toxic patients treated with amiodarone. ${ }^{15,17-18}$ Despite the fact that the exact mechanism of amiodarone-toxicity has not yet been elucidated, the association of foamy AM accumulation with amiodarone and other agents that have caused clinically significant pulmonary toxicity raises questions about the safety implications of the AM response observed following inhalation of PS AONs. The purpose of this study was to investigate impact of exposure of AMs to AONs with regard to AM morphology and function, with the further aim of understanding the toxicologic significance of the foamy AM response to inhaled AONs. We developed an in vitro model of foamy macrophages using RAW 264.7 macrophage cells. RAW 264.7 cells have been used previously as in vitro models of AM for responses to asbestos, ${ }^{19}$ airborne particulate matter, ${ }^{20}$ air pollution ${ }^{21}$ and LPS-induced lung injury. ${ }^{22}$ In our model, the morphological and functional changes following exposure to AONs were characterized and compared to the effects of amiodarone.

\section{Materials and Methods}

\section{Cell line and reagents}

The RAW 264.7 murine macrophage cell line and High Glucose Dulbeco's Modified Eagle's Medium (DMEM) were obtained from ATCC (Manassas, VA, USA). Amiodarone, Nile Red and cytochalasin D were obtained from SigmaAldrich (St. Louis, Missouri, USA), while phosphate-buffered saline (PBS), trypsin-EDTA and fetal bovine serum (FBS) were obtained from Hyclone (Logan, Utah, USA), 1-um yellowgreen carboxylate-modified fluorescent FluoSpheres ${ }^{\circledR}$ were procured from Invitrogen (Carlsbad, California, USA), and Bovine Serum Albumin fraction V (BSA) was purchased from EMD Biosciences (Gibbstown, New Jersey, USA). The two AONs (a 1:1 mixture) used in this study were antisense sequences targeting rat genes; one the common beta chain of the IL-3, IL-5 and GM-CSF receptor and the other CCR3, which are the same targets (but not sequences) as the human asthma drug, PXS TPI ASM8. Both were 18 nucleotides in length (18-mer) and were manufactured by Girindus (Cincinnati, $\mathrm{OH}$, USA). The sequences are TGGCACTTTAGGTGGCTG (TOP006) and ACTCATATTCATAGGGTG (TOP007). These AONs contained a full-length PS backbone, as is commonly used for AON drug candidates, to confer resistance to endogenous nucleases.

\section{Cell maintenance and drug-treat- ment}

RAW 264.7 cells were maintained in DMEM, supplemented with $10 \% \mathrm{FBS}$ at $37^{\circ} \mathrm{C}$ in a $5 \%$ $\mathrm{CO}_{2}$ humidified incubator. The day before drug treatment, $7.5 \times 10^{4}$ cells/well were seeded in 24-well plates. The cells were then exposed to various concentrations $(0.5$ to $30 \mu \mathrm{M})$ of either amiodarone or AON for $48 \mathrm{~h}$.

\section{Intratracheal delivery of oligonu- cleotides to mice}

Male CD-1 mice, weighing between $23-25 \mathrm{~g}$ (Charles River Laboratory, Saint Constant, QC, Canada), were anesthetized with isoflurane, intubated and administered with AONs prepared in sterile (RNAse-DNAse free) water at concentrations such that a volume of $25 \mu \mathrm{L}$ administred intratracheally using a microsprayer (PennCentury,Wyndmoor, PA, USA) would provide a dose of either $0.75 \mathrm{mg} / \mathrm{kg}$ or $2.5 \mathrm{mg} / \mathrm{kg}$. Mice received 10 treatments of AON on weekdays. 24 hours after the last AON treatment, mice were sacrificed, exsanguinated and BAL was performed by injecting and retrieving $1 \mathrm{~mL}$ of cold PBS supplemented with $0.1 \mathrm{mM}$ EDTA 5 times into lungs of mice. BAL was collected and centrifuged to collect cells. Cytospins of BAL cells were stained with Hema-3 (Fisher Scientific) for assessment by a pathologist.

\section{Morphology and electron microscopy}

For light microscopy, drug-treated cells were collected, counted using the GUAVA-Viacount reagent (Millipore, Billerica, MA, USA), and 5 $\times 10^{4}$ cells were transferred onto slides and stained with Hema-3 Quick Stain. Slides were examined by Dr. Groom of CanBioPharma Consulting Inc, a pathologist with experience viewing lungs of mice treated with ONs. For tandem electron microscopy analysis, cells were prepared as described previously. ${ }^{23}$ Briefly, adherent drug-treated cells were fixed in $2.5 \%$ glutaraldehyde / $0.1 \mathrm{M}$ sodium cacodylate buffer, dehydrated and embedded in lowviscosity, thermally-curing Epon resin. Ultrathin sections $(70-80 \mathrm{~nm})$ were cut from the resin blocks by using a Reichert-Jung Ultracut E ultramicrotome with a Diatome (Biel, Switzerland) diamond knife. The sections were transferred to 300-mesh formvar $\mathrm{Cu}$ TEM grids for image analysis in a JEOL JEM2011 TEM equipped with a Gatan (Pleasanton, CA, USA) $300 \mathrm{~W}$ charge-coupled device camera.

\section{Nile red staining (for lipid content)}

Following treatment, cells were collected, counted, washed with PBS and then resuspended at $6 \times 10^{5}$ cells $/ \mathrm{mL}$ in a $1.5 \mu \mathrm{g} / \mathrm{mL}$ Nile Red solution. The fluorescence of the stained cells was evaluated after a 20 min incubation period using the GUAVA EasyCyte microcapillary flow cytometry system. Data analysis was performed using the FCS Express software.

\section{Assessment of cell viability and apoptosis}

Following treatment, cells were collected and stained with GUAVA-Viacount reagent, allowing viable and apoptotic cell numbers to be quantified with the GUAVA EasyCyte microcapillary flow cytometry system.

\section{Cytokine profiling}

Levels of IP-10, IL-1RA, IL-6, TGF- $\beta 1$, IL-1 $\beta$, MIP- $1 \alpha$ and TNF- $\alpha$ were assessed in cell supernatants by SearchLight Multiplex Immunoassay (Aushon BioSystems, Billerica, MA, USA).

\section{Phagocytosis assay}

To facilitate microsphere uptake, carboxylated polystyrene $1 \mu \mathrm{m}$ yellow-green microspheres were coated with BSA $\left(10 \mathrm{mg} / \mathrm{mL}, 37^{\circ} \mathrm{C}, 60\right.$ min), washed in PBS, resuspended at a concentration of $1.5 \times 10^{8}$ microspheres/mL in PBS, and sonicated prior to use. These BSA-coated microspheres $\left(\sim 1.5 \times 10^{5} /\right.$ well $)$ were added to the cells, and plates were centrifuged at $250 \mathrm{~g}$ for $10 \mathrm{~min}$. Cells were harvested at different time-points following incubation at $37^{\circ} \mathrm{C}$, washed and resuspended in 4\% paraformaldehyde in PBS before being analyzed by flow cytometry. As a control for non-phagocytic uptake, cells were pre-treated with the phagocytosis inhibitor cytochalasin D $(5 \mu \mathrm{g} / \mathrm{mL}$, $\left.37^{\circ} \mathrm{C}, 60 \mathrm{~min}\right)$.

\section{Statistical tests}

Direct comparison of Nile red uptake by amiodarone treated cells with AON-treated cells at each concentration was performed using a t-test. For comparison of the effect of either amiodarone or AON on cell viability, cytokine production and phagocytosis, an ANOVA was performed with a Dunnett's using the respective vehicle-treatment control group.

\section{Results}

\section{Morphological changes and phos- pholipid accumulation following drug treatment}

To characterize the potential effects of AON exposure on macrophages, an in vitro model of foamy AM was established. Murine macrophages (RAW 264.7) cells were exposed to amiodarone, a known inducer of foamy AM as a comparator drug, for $48 \mathrm{~h}$, and both morphological and functional responses of the macrophages were then assessed. Following $48 \mathrm{~h}$ exposure to amiodarone at concentrations $\leq 5 \mu \mathrm{M}$, no morphological changes were evident (Figure 1C and Table 1), whereas at concentrations $>5 \mu \mathrm{M}$, cytoplasmic vacuolation 
Table 1. Findings (\%) of cytologic phenotype of macrophages in response to treatment.

\begin{tabular}{|c|c|c|c|c|c|c|c|c|c|c|c|c|c|c|c|}
\hline \multirow{2}{*}{$\begin{array}{l}\text { Cell type } \\
\text { Treatment } \\
\text { Concentration/dose }\end{array}$} & \multicolumn{6}{|c|}{ RAW 264.7 cells } & & \multicolumn{5}{|c|}{ AON $(\mu \mathrm{M})$} & \multicolumn{3}{|c|}{$\begin{array}{c}\text { BAL of mice } \\
\text { Antisense } \\
\text { (mg/kg per day) }\end{array}$} \\
\hline & $\begin{array}{c}\text { Vehicle } \\
\text { (DMSO) } \\
n=2\end{array}$ & 5 & 10 & 15 & 20 & $\begin{array}{l}\text { Vehicle } \\
\text { (PBS) }\end{array}$ & 5 & 10 & 15 & 20 & 25 & 30 & $\begin{array}{c}\text { Vehicle } \\
\text { (PBS) } \\
n=5\end{array}$ & 0.75 & 2.5 \\
\hline
\end{tabular}

Cytoplasmic vacuolation

minimal

100

$\begin{array}{ccccccccc}- & - & - & 25 & 33 & 75 & 75 & 75 & 75 \\ 100 & - & - & - & 33 & 25 & 25 & 25 & 25\end{array}$

$100 \quad 14$

mild 100

moderate

Cytomegaly

minimal

mild

moderate

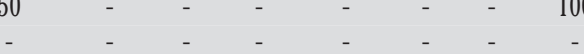

marked

n, number of slides assessed; AON, Antisense oligonucleotides.

was noted (Figure $1 \mathrm{E}$ and $1 \mathrm{G}$ and Table 1). The vacuolation was characterized by the presence of either one large irregular vacuole in the cytoplasm of cells or multiple small round vacuoles that distended the cytoplasm of the cells. At amiodarone concentrations above $5 \mu \mathrm{M}$, the average histologic severity grade increased progressing from minimal to moderate as the concentration of amiodarone increased (Table 1). In comparison, $48 \mathrm{~h}$ incubation of cells with $5 \mu \mathrm{M}$ AON resulted in minimal vacuolation (Table 1), and the severity grade for the vacuolization increased only to a level of mild even at the highest AON concentration (30 $\mu \mathrm{M}$, Figure 1 and Table 1).

Cytospins of the BAL fluid collected from mice whose lungs were exposed to the same AON used above in the in vitro studies but for 10 repeated treatments showed similar vacuolation of AMs (Table 1). Specifically, doses of 0.75 or $2.5 \mathrm{mg} / \mathrm{kg} /$ day resulted in findings of minimal and mild AM vacuolation, respectively (Table 1). The cytological appearances of macrophages in the BAL were similar to the histologic appearance of macrophages accumulating in the alveolar lumens observed histologically in lungs from mice exposed to AON (data not shown). Additional findings of cytoplasmic basophilia (not shown) and higher severity of cytomegaly were observed in BAL of mice as compared to RAW 264.7 cells, demonstrating a limitation of the in vitro model. However, with regard to macrophage vacuolation, the vacuolation observed with in vitro exposure of mouse macrophages is considered to be phenotypically similar to the AM vacuolation that occurs with in vivo inhalation administration of PS AONs.

To further characterize the morphological changes, electron microscopy was performed to determine whether the vacuoles exhibited a lamellated substructure that would be suggestive of phospholipid content (i.e., lamellar whorls). Electron microscopic examination revealed that, following treatment with amiodarone ( $7 \mu \mathrm{M}$, Figure $2 \mathrm{~B}$ and $2 \mathrm{E})$, the cells had
A.

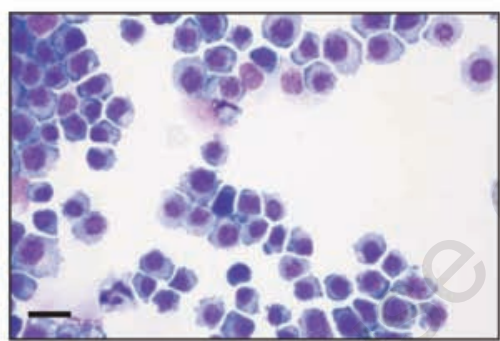

c.

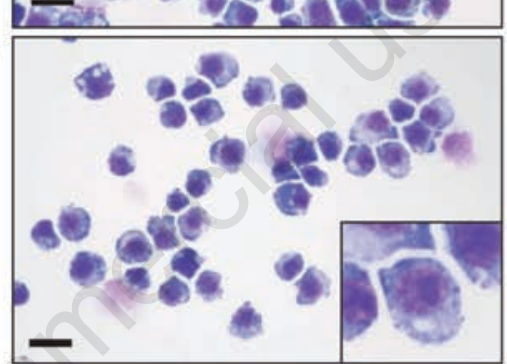

E.

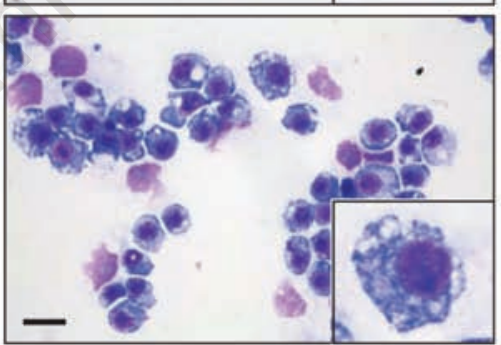

G.

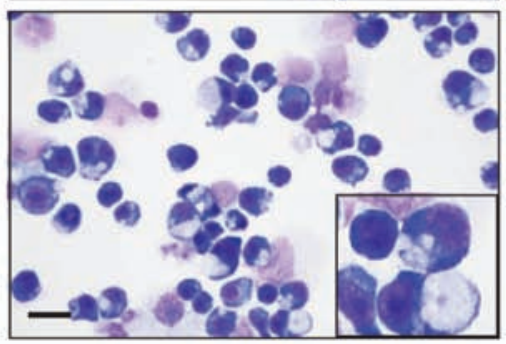

Figure 1. Morphological changes in response to treatments. Representative light micrographs (63X) of RAW 264.7 cells treated with amiodarone vehicle $A$ ), amiodarone at 5,15 , $20 \mu \mathrm{M}$ final $\mathrm{C}, \mathrm{E}, \mathrm{G})$, antisense oligonucleotides (AON) vehicle B) or AON at 5, 15, $20,30 \mu \mathrm{M}$ final $\mathrm{D}, \mathrm{F}, \mathrm{H}, \mathrm{I})$. The bar represents $20 \mu \mathrm{m}$. Insert is an enlargement $(2.5 \mathrm{X})$ of a selected region using Photoshop Illustrator.

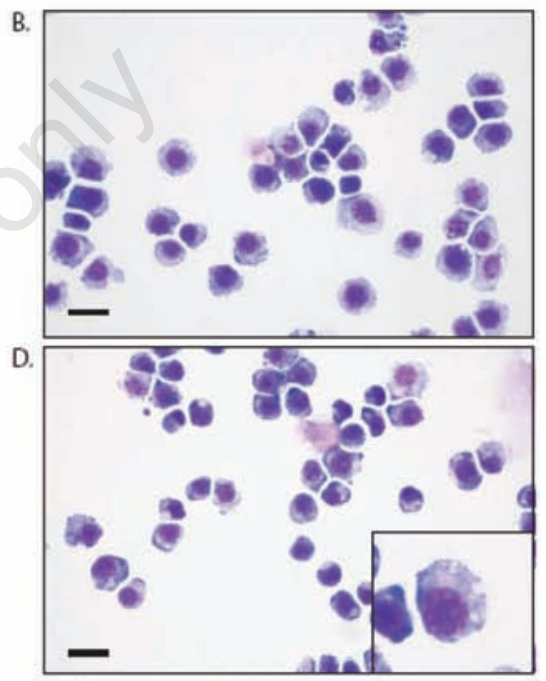

F.
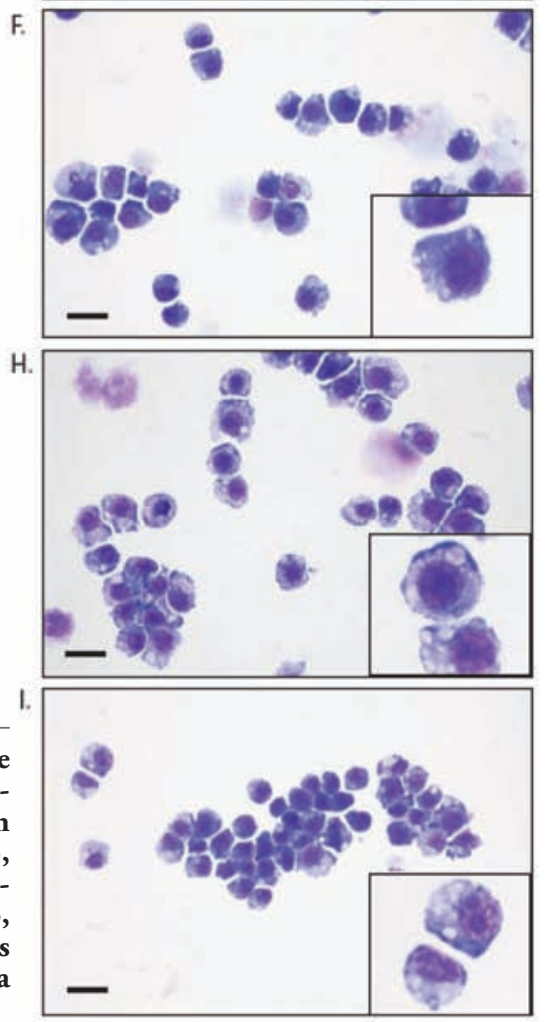
vacuoles containing material with lamellar structures characteristic of phospholipids, and these lamellar vacuoles were absent in control cells (Figure 2A and 2D). In electron micrographs of AON-treated cells $(7 \mu \mathrm{M}$, Figure $2 \mathrm{C}$ and $2 \mathrm{~F}$ ), the vacuoles also exhibited a lamellar substructure similar to the appearance of amiodarone-treated cells (Figure 2B/2E).

Nile Red staining was employed to confirm the presence of intracellular phospholipids. ${ }^{24}$ In amiodarone-treated cells, a dose-dependent accumulation of phospholipids was observed, starting at $2.5 \mu \mathrm{M}$ and increasing through 17.5 $\mu \mathrm{M}$, at which concentration, there was a mean increase of $103 \pm 7 \%$ in Nile Red staining, compared to levels in vehicle-treated cells (Figure 3). Treatment with AON also produced an increase in Nile Red staining at $\leq 2.5 \mu \mathrm{M}$, and the percent change observed at $2.5 \mu \mathrm{M}(29 \pm$ 7\%) was similar to that observed with an equimolar concentration of amiodarone $(38 \pm 6 \%)$ (Figure 3). However, from this initial increase in Nile Red staining for the AON, the level then remained unchanged with increasing AON concentrations (Figure 3), such that at the highest concentration tested for both agents $(17.5 \mu \mathrm{M})$, there was more than a 3 -fold difference in Nile Red staining between amiodarone- and AON-treated cells (Figure 3).

\section{Cell viability and apoptosis follow- ing drug treatment}

Along with changes in cellular morphology and lipid accumulation, changes in cell survival were assessed. In amiodarone-treated cells (48 $\mathrm{h}$ incubation), a significant reduction in cell number was observed, starting at an amiodarone concentration of $10 \mu \mathrm{M}$ (Figure $4 \mathrm{~A})$, and this decrease in cell numbers continued until only $26 \%(\mathrm{P}<0.001)$ of total cells remained following exposure to $20 \mu \mathrm{M}$ of amiodarone (Figure 4A). Of those remaining cells, fewer than $25 \%(21 \pm 8 \% ; \mathrm{P}<0.01)$ were viable (Figure 4B). Furthermore, amiodarone treatment resulted in an increased percentage of apoptotic cells of up to $47 \pm 1 \%$ at the highest concentration $(\mathrm{P}<0.01)$ (Figure $4 \mathrm{~B})$. In contrast, $48 \mathrm{~h}$ incubation of cells with AON at concentrations up to $25 \mu \mathrm{M}$ did not reduce total cell counts, did not decrease cell viability (Figure 4B), or increase the number of apoptotic cells.

\section{Cytokine production in amio- darone- and AON-treated cells}

Functional responses of the cells were also investigated, including measurement of cytokine levels in culture supernatants of treated cells. TNF- $\alpha$ levels significantly increased in response to amiodarone and AON, as compared to vehicle-treated cells $(362 \pm 41$ $\mathrm{pg} / \mathrm{mL}$ for amiodarone vehicle and $327 \pm 30$ $\mathrm{pg} / \mathrm{mL}$ for AON vehicle Figure $5 \mathrm{~A}$ ). The mean
A

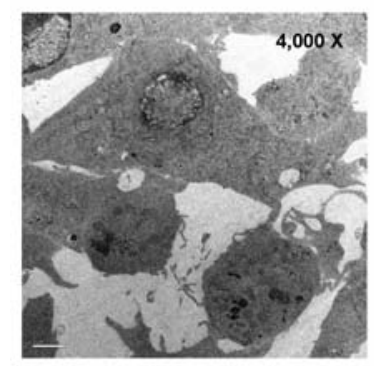

D

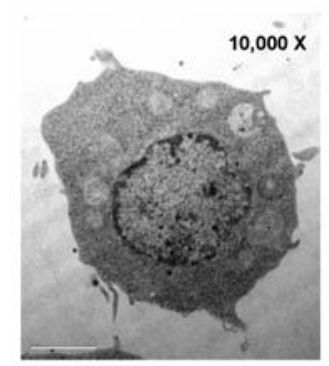

B

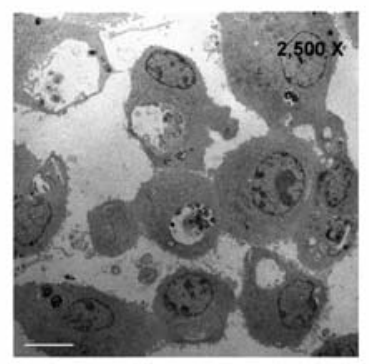

E

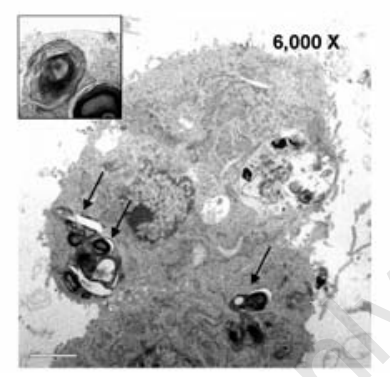

C

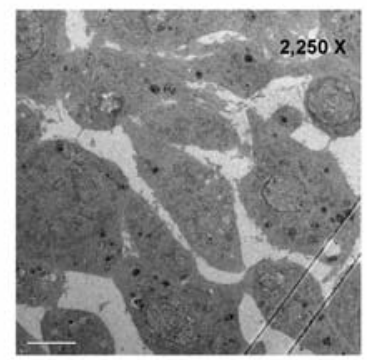

F

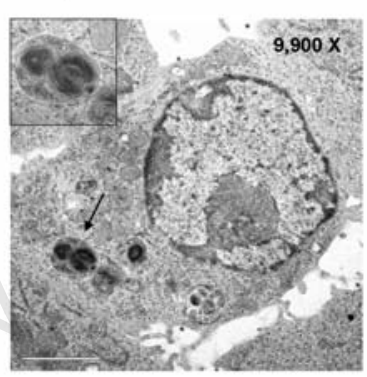

Figure 2. Accumulation of intracellular lamellar bodies following treatment. Following $48 \mathrm{~h}$ treatment with vehicle A, D), amiodarone B, E) or antisense oligonucleotides (AON) $\mathrm{C}$, F) at $7 \mu \mathrm{M}$, cell morphology was examined by TEM. Magnifications are indicated in the top right corner of each image.

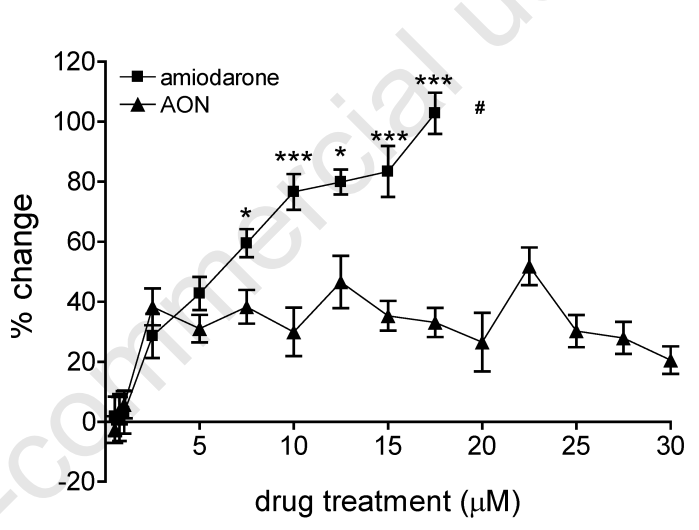

Figure 3. Intracellular phospholipid accumulation following treatment. Treated RAW 264.7 cells were stained with Nile Red, and fluorescence was assessed. The percent change in red fluorescence vs. vehicle (mean \pm SEM) is shown $(n=3-15)$. Ttests of the data for amiodarone vs. antisense oligonucleotides (AON) at each drug concentration are represented as follows: ${ }^{*} \mathbf{P}<0.05 ;{ }^{* * *} \mathbf{P}<0.001$. " At higher amiodarone concentrations, cell death was observed, preventing analysis.
A

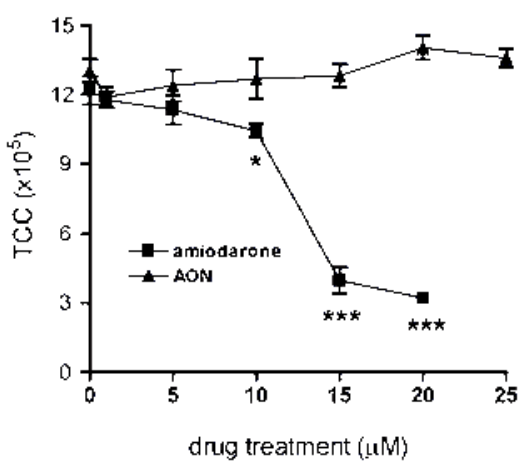

B

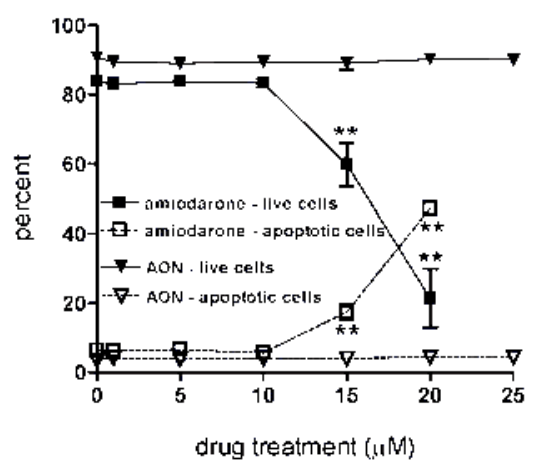

Figure 4. Effect of amiodarone and AON on cell viability. Following $48 \mathrm{~h}$ treatment with drugs, cell counts and viability were determined. Mean total cell counts \pm SEM (TCC) $(A), n=3-15$, and mean percent viability \pm SEM $(B), n=6-15$, are shown. ANOVA with Dunnett's vs. vehicle: ${ }^{*} \mathbf{P}<0.05 ;{ }^{* *} \mathbf{P}<0.01{ }^{* * *} \mathbf{P}<0.001$. 
induction in response to $15 \mu \mathrm{M}$ of amiodarone reached a maximum of $2320 \pm 298 \mathrm{pg} / \mathrm{mL}$ for a 5.4 fold increase over the respective vehicle mean level, and approximately 2.8-fold the level of TNF- $\alpha(614 \pm 79 \mathrm{pg} / \mathrm{mL})$ produced by incubation with a $30 \mu \mathrm{M}$ concentration of AON (twice the concentration of amiodarone that was tested). Mean MIP-1 $\alpha$ levels also significantly increased with increasing amiodarone concentrations, with maximum mean values reaching $232 \pm 19 \mathrm{ng} / \mathrm{mL}$ in response to $15 \mu \mathrm{M}$ amiodarone, which was greater than 6 times the respective vehicle mean level (Figure 5B). Increasing the concentration of amiodarone to $20 \mu \mathrm{M}$ resulted in a sharp decrease in MIP-1 $\alpha$ levels (Figure 5B), which may be secondary to the above described cytotoxicity of amiodarone. As observed for TNF- $\alpha$, in response to a concentration of AON $(30 \mu \mathrm{M})$ twice that of amiodarone, mean MIP-1 $\alpha$ levels peaked at only 2 -fold above the respective mean for vehicle-treated cells $(78 \pm 17 \mathrm{ng} / \mathrm{mL} ; \mathrm{P}<0.01$ compared to vehicle). For IL-1RA, a maximum mean level of $29.1 \pm 3.6 \mathrm{ng} / \mathrm{mL}$ was reached at the highest concentration of amiodarone (20 $\mu \mathrm{M}$, Figure $5 \mathrm{C}$ ), while in response to $\mathrm{AON}$, the IL-1RA levels remained near baseline values $(1.6 \pm 0.8 \mathrm{ng} / \mathrm{mL})$ throughout the concentration range tested. In contrast to the aforementioned cytokines, IP-10 levels decreased with increasing amiodarone concentration, such that in response to $20 \mu \mathrm{M}$ amiodarone, mean IP-10 levels were only $15 \%$ of those treated with vehicle (Figure 5D). AON exposure resulted in an initial spike in IP-10 levels $(4553 \pm 218 \mathrm{pg} / \mathrm{mL}$ at $2.5 \mu \mathrm{M} \mathrm{ON} ; \mathrm{P}<0.01)$, after which the overall levels of IP-10 gradually decreased, reaching $1426 \pm 115 \mathrm{pg} / \mathrm{mL}$ at $30 \mu \mathrm{M}$ (Figure 5D). IL-10 levels decreased from a baseline of $26 \pm 3 \mathrm{pg} / \mathrm{mL}$ in vehicle control cells to $1.0 \pm 0.7 \mathrm{pg} / \mathrm{mL}$ in response to the highest concentration of amiodarone $(20 \mu \mathrm{M}$, Figure $5 \mathrm{E})$, whereas an equimolar concentration of AON resulted in a decrease of IL-10 to $9.9 \pm 1.2$ $\mathrm{pg} / \mathrm{mL}$, and the levels remained steady even at $30 \mu \mathrm{M}$ of AON. Finally, IL-6, TGF- $\beta 1$ and IL- $1 \beta$ levels were similar to vehicle treated levels for all amiodarone and AON concentrations tested (data not shown).

\section{Effect of amiodarone and AON treatment on the RAW 264.7 phagocytic activity}

An additional functional response, the phagocytosis capacity of macrophages, was also investigated. Phagocytosis was assessed by incubating treated cells with BSA-coated fluorescent microspheres ( $1 \mu \mathrm{m}$ in diameter) and measuring cell uptake over time. To avoid interference by the increased number of apoptotic cells in response to high concentrations of amiodarone, only live cells were included in this analysis. Microsphere uptake, as revealed by the increase in the mean fluorescence intensity (MFI), started in vehicle control cells (DMSO-treated) after 60 min of incubation and remained at this level for the next $2 \mathrm{~h}$ (180 min total) (Figure 6A). This uptake (change in MFI) could be significantly decreased by pre-treating cells with the phagocytosis inhibitor cytochalasin D (75\% inhibition versus vehicle-treated cells, $\mathrm{P}<0.01$ ) (Figure 6), even when cells were incubated with the microspheres for up to $180 \mathrm{~min}$. Treatment with a low concentration of amiodarone $(1 \mu \mathrm{M})$, did not alter microsphere uptake, as compared to vehicle-treated cells. At a 10-fold higher amiodarone concentration $(10 \mu \mathrm{M})$, no effect on phagocytosis at 60 min was observed but a modest yet significant increase in phagocytosis was evident at 180 min (27\% increase versus vehicle-treated cells, $\mathrm{P}<0.05$, Figure. $6 \mathrm{~A})$. However, further increasing the concentration of amiodarone to $15 \mu \mathrm{M}$ resulted in a significant decrease in the ability of the cells to phagocytose the microspheres, with $84 \%(\mathrm{P}<0.01)$ and $65 \%(\mathrm{P}<0.01)$ reduction in the uptake at 60 and $120 \mathrm{~min}$. This level of inhibition was similar to that measured in cells pre-treated with cytochalasin D (Figure 6A). However, when the incubation time with the microspheres was extended to $180 \mathrm{~min}$, the phagocytosis activity returned to the levels of the control cells. Phagocytosis could not be assayed in cells treated with higher concentrations of amiodarone, as cell death was too excessive (data not shown). Following
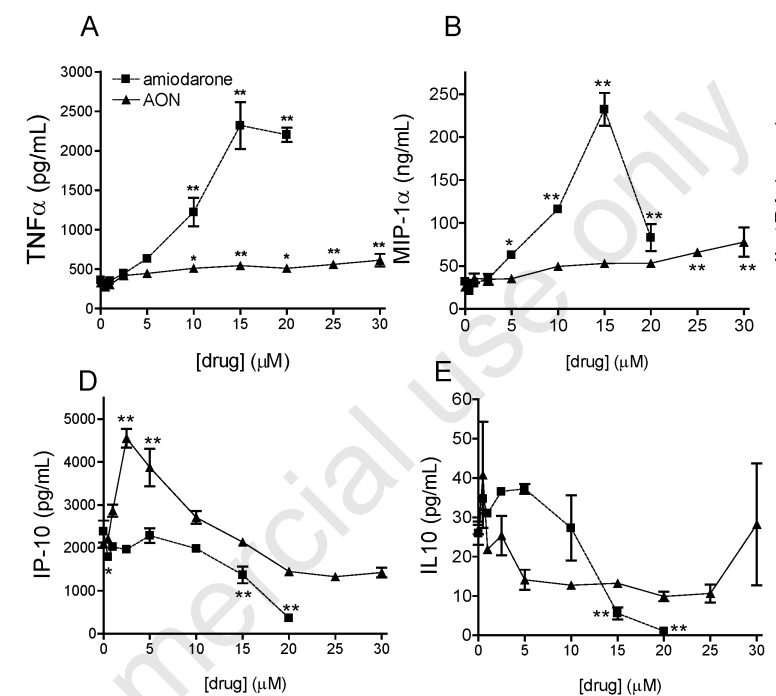

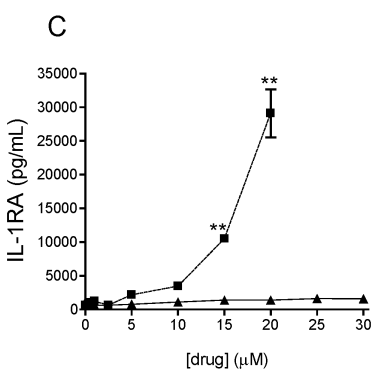

Figure 5. Inflammatory mediator release. Cell culture supernatants were harvested following the drug treatment. Levels of TNF $-\alpha$ (A), MIP-1 $\alpha$ (B), IL 1RA (C), IP 10 (D) and IL-10 (E) were measured by ELISA. Mean $\mathrm{pg} / \mathrm{mL} \pm$ SEM are shown $(\mathrm{n}=3)$. ANOVA with Dunnett's vs. vehicle: ${ }^{*} \mathbf{P}<0.05$; $^{* *} \mathbf{P}<0.01$.
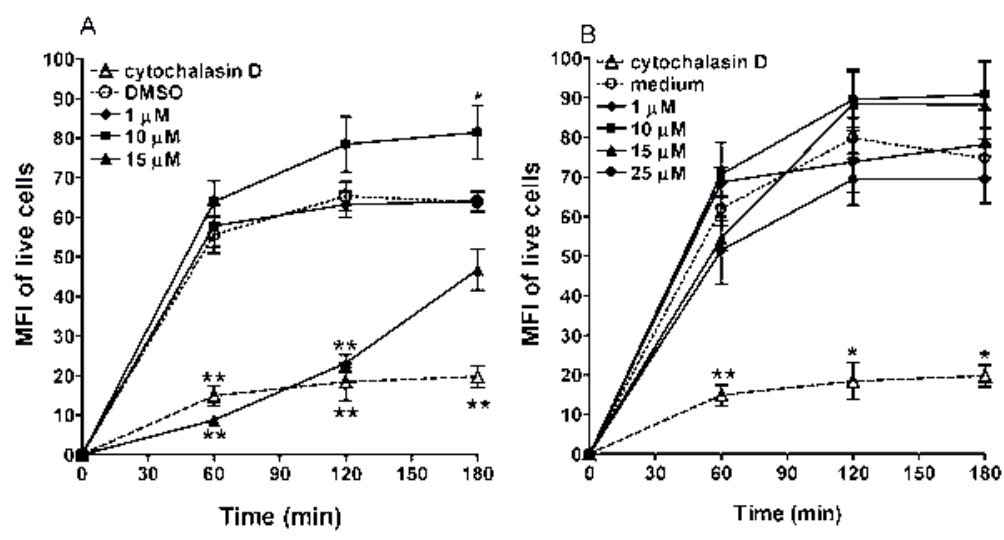

Figure 6. Phagocytic activity of RAW 264.7 cells. Following treatment with amiodarone, its vehicle (DMSO) (A), AON or its vehicle (media alone) (B), cells were incubated with BSA-coated fluorescent microspheres for 60,120 or $180 \mathrm{~min}$. Mean fluorescence intensities (MFI) of gated live cells (mean \pm SEM) are shown $(n=6-12)$. A cell preparation pretreated with the phagocytosis inhibitor, cytochalasin $\mathrm{D}$, was included as a positive control. ANOVA with Dunnett's vs. vehicle for each time point: ${ }^{*} \mathbf{P}<0.05$; ${ }^{* *} \mathbf{P}<0.01$. 
AON treatment at concentrations ranging from 1 to $25 \mu \mathrm{M}$, no change in phagocytosis was observed, based on the absence of any apparent change in fluorescence levels relative to the vehicle-treated cells. The results indicate that AON treatment, in contrast to amiodarone, did not alter the phagocytic activity of RAW 264.7 macrophages (Figure 6B).

\section{Discussion}

Since the concept was proposed by Zamecnik and Stephenson ${ }^{25}$ to use antisense AONs to modulate expression of a target gene by binding to its mRNA and preventing translation, there have been concerted efforts to develop AONs as drug therapeutics for a wide array of indications, including respiratory diseases. One of the key challenges to the development of any drug is to achieve effective tissue concentrations without eliciting adverse effects. In animals, following systemic delivery of PS AONs, blood-level related manifestations of toxicity have been observed (i.e., activation of the alternative complement pathway and inhibition of the intrinsic coagulation cascade) ${ }^{26-27}$ as well as changes in the major organs of AON accumulation such as kidneys, liver and spleen. ${ }^{28-29}$ Observations in the organs include mononuclear cell infiltrates and endosomal accumulation of the AON in the form of basophilic granules within resident macrophages of the spleen, lymph nodes and liver (Kupffer cells) and within the proximal tubule cells of the kidney. ${ }^{30}$

The lung represents a unique target tissue organ for AON-based therapy, as it is readily accessible by direct and non-invasive delivery to the site of action (via oral inhalation). Topical delivery should minimize systemic exposure $^{1}$ and therefore significantly reduce the risk for adverse effects on internal organs, as well as the known blood-level-related toxicities described above. However, similar to what has been observed in other tissue sites of AON uptake, inhalation of AON can lead to local cellular infiltration, typically observed in the interstitium and elsewhere in the lung parenchyma. This is mainly a perivascular infiltrate and is not observed in the alveolar space. However, a distinctly different type of change in the lungs is commonly observed following repeated inhalation administration of PS AONs, which is accumulation of macrophages in the alveoli (i.e., AM) that often contain intra-cytoplasmic basophilic granular material, possibly reflecting uptake of the AON (i.e., akin to basophilic granulation observed in other tissues of AON deposition).$^{2,9}$ Because of this granular appearance, the AMs have been described as foamy, although the more common use of this term has been in association with lipid accumulation within AMs caused by other agents or the accumulation of noxious particulate matter.

In addition to their critical role in particulate clearance and host defenses, the AM is an important cell of the alveolar space, as it is able to release immunologic or toxic mediators. In the lung, dramatic migration of macrophages into the alveolar space has been a concern, as histological evidence from both human ${ }^{12}$ and animal $^{13}$ models of pulmonary fibrosis suggests a relationship between the fibrotic changes and the abundance of macrophages appearing vacuolated or foamy, although the precise mechanism by which these cells contribute to fibrotic alterations is not fully understood. In general, pulmonary fibrosis is believed to result from the dysregulation of wound repair, which arises from inflammatory cell influx and release of pro-fibrotic mediators. $^{31-32}$ Such mediators include various chemokines, cytokines and growth factors, which, if sufficiently induced, can alter the balance of cell proliferation and turn the healing response into a fibrotic response. . $^{31,33}$ Therefore, for any agent administered by inhalation that induces an influx of AMs into the alveolar space, questions may arise as to whether this influx may have pro-inflammatory consequences or by some other means poses a risk of lung injury that could lead to fibrotic changes. Hence, this is one concern about the AM response to inhaled AONs. In addition, the vacuolated or granular appearance of the cells raises questions about the content of the cellular inclusions and whether the infiltrating AMs may be activated, or conversely, may have sustained a pertubation of their normal function.

An example of an agent that induces the accumulation of foamy AMs is the antiarrhythmic drug, amiodarone. Oral administration of this drug is associated with serious pulmonary toxicity (reviwed in Papiris et al. ${ }^{16}$ ) which can lead to its discontinuation in patient treatment. Lung biopsies from patients with amiodarone-induced pulmonary toxicity revealed several types of changes including an abundance of foamy or vacuolated AMs. These vacuoles include both small optically empty vacuoles and large phagolysosomes containing phospholipid material organized into lamellar structures as viewed by electron microscopy. ${ }^{16}$ While some studies have described the response to amiodarone with the pulmonary histology characterized by profuse infiltration of foamy AMs (containing numerous vacuoles) and fibrotic changes that impair pulmonary function $^{12-13}$ it is unclear whether the accumulation of foamy macrophages is a direct cause of the pulmonary fibrosis. As demonstrated in one placebo-controlled study with amiodarone in patients with congestive heart failure, severe pulmonary fibrosis was encountered in $1.2 \%$ of patients receiving amiodarone as compared to $0.9 \%$ in the control group. ${ }^{34}$ Nevertheless, owing to the legacy of the experience with amiodarone and other similar pulmonary toxicants that elicit AM infiltration as part of their impact on the lungs, inferences have been drawn about the possible risk for lung damage associated with AON-induced AM accumulation.

To investigate the morphologic changes and functional consequence of the AM response to $\mathrm{AON}$, we developed an in vitro model of foamy macrophages using murine macrophages (RAW 264.7 cells) and amiodarone as a positive control drug. Our results showed that RAW 264.7 cells acquired a vacuolated or foamy appearance in response to amiodarone, which increased in severity grade with increasing concentration of amiodarone. Cells exposed to a PS AON had a similar vacuolated appearance, which was noted at a lower molar concentration of AON $(5 \mu \mathrm{M})$ than the minimal vacuoleinducing concentration of amiodarone. However, the severity grade of vacuolation in response to increasing concentrations of the AON did not reach the level of that observed with amiodarone. Cytomegaly, another finding, was also observed in cells exposed to amiodarone $(20 \mu \mathrm{M})$ or the PS AON $(30 \mu \mathrm{M})$.

To directly compare observations from cell lines to in vivo findings is challenging, especially when accounting for differences in the preparations (cytospins of in vitro cell cultures vs histopathology slides). To this end, we opted to perform cytospins of BAL fluid harvested from mice that had received repeated intratracheal doses of AON (for 10 consecutive days). This repeated dosing mimicked the multiple delivery of AON that would occur in patients, and the cytospin preparations were similar to the preparations used for the cell lines. The cytospin preparations of BAL from AON-treated but not vehicle control animals confirmed the presence of vacuolated macrophages and cytomegaly, thereby validating the potential of the in vitro model to produce morphologic changes analogous to in vivo administration. Noteworthy is the absence of cytoplasmic basophilia in the in vitro model. As the BAL findings were observed after 10 repeated doses, it is unclear whether the absence in vitro reflects either a time or dose limitation of this model or whether certain in vivo aspects can not be replicated in vitro.

Further characterization of the vacuolated macrophages using electron microscopy demonstrated the presence of characteristic lamellated cytoplasmic inclusions in cells exposed to either amiodarone or PS AON. To quantify phospholipid accumulation, Nile Red staining was used. A similar intracellular level of phospholipid was observed in response to treatment with amiodarone or PS AON at an 
equimolar concentration of $2.5 \mu \mathrm{M}$. However, at concentrations $>5 \mu \mathrm{M}$, the phospholipid accumulation in response to amiodarone continued to increase, while the levels in AON treated cells remained constant, suggesting a clear difference in the responses of the cells, although their morphologies appeared similar. Amiodarone-induced phospholipidosis has been linked to the inhibition of lysosomal phospholipase A2 activity in Madin-Darby canine kidney cells. ${ }^{35}$ Future biochemical studies may determine whether AON also interfere with lysosomal phospholipase A2 activity albeit at a lower degree than amiodarone. Our findings of phospholipid accumulation and the presence of lamellar bodies in amiodaronetreated macrophages are consistent with those changes reported in rat and human hepatocytes ex vivo ${ }^{36}$ demonstrating the utility of the in vitro model.

In our model, amiodarone treatment significantly affected the viability and induced apoptosis of the cells, results in line with previous reports ${ }^{37}$ while treatment with the AON did not affect cell viability nor induce apoptosis at the highest concentration tested $(30 \mu \mathrm{M})$, although these cells did have the vacuolated or foamy appearance.

Functional consequences of drug treatment were assessed in terms of the secretion of cytokines, as typically, inflammation preceeds the development of fibrosis. ${ }^{33}$ Amiodaronetreated macrophages secreted high levels of the pro-inflammatory chemokines/cytokines MIP-1 $\alpha$, IL 1RA and TNF- $\alpha$, but levels of IP-10, a cytokine with protective properties, ${ }^{38}$ were decreased In contrast, AON exposure did not alter the levels of IL-1 RA, resulted in slight increases in TNF- $\alpha$, and only increased MIP$1 \alpha$ levels approximately 2 -fold. Exposure of the cells to a low AON concentration resulted in IP10 production, which then returned to levels similar to baseline as the concentration of AON was increased. These results again highlight that, although both drug treatments produced similar morphological changes in the cells, the cytokine secretion responses of the cells were highly dissimilar. While neither amiodarone nor AON treatment altered the levels of TGF-beta, a cytokine associated with fibrosis, we can not exclude that the absence of the cytokine may reflect the limitation of the in vitro model with its $48 \mathrm{~h}$ exposure time.

Others have shown no impairment of pulmonary host defence mechanisms in the rat, ${ }^{39}$ nor changes in the phagocytosis of heat-killed yeast cells ${ }^{39}$ by AM with a foamy appearance. Results from our model differ from these other studies in that we showed that amiodarone significantly impaired the ability of the macrophages to phagocytose microspheres. When sufficient time was given, phagocytosis returned to control levels, reflecting a tran- sient response and not a prolonged suppression of phagocytosis by amiodarone, which may explain the discrepancy between our findings and those of others. Chemotaxis responses of the treated cells was not measured in our system, but it is possible that amiodarone treatment perturbed the motility of the cells, resulting in a temporary reduction of phagocytosis capability. Although the mechanism is unclear, the observation is again that differing from the response to amiodarone, treatment of macrophages with PS AON had no effect on the ability of macrophages to phagocytose foreign material (BSA-coated microspheres).

These differences in responses to amiodarone and the PS AON could be explained, in part, by the different physiochemical properties of these molecules. Amiodarone is an amphiphilic drug that exhibits characteristic and distinct pulmonary effects. ${ }^{40}$ In animals receiving such drugs, foamy cells have been shown to develop as a result of the impairment of the metabolism of phospholipids within the cell. ${ }^{14,35}$ AONs are polyanionic molecules, and their impact on macrophages following uptake may differ from that of amiodarone and related drugs. Along with future work to determine the mechanism of the foamy appearance, work needs to include studies in human monocytes and macrophages or if possible human AM. To date, delivery of inhaled AON to humans has not resulted in increased macrophage numbers recovered from induced sputum, ${ }^{41}$ but further biochemical characterization of these macrophages has yet to be undertaken.

In summary, exposure of macrophages to a PS AON in vitro resulted in a vacuolated or "foamy" appearance, which was characterized by the presence of intracellular lamellated structures and an accumulation of phospholipids, changes that were qualitatively similar to those induced by amiodarone. However, in contrast to amiodarone, the AON-treated cells did not exhibit functional changes with respect to viability, apoptosis or with functions that can be regarded as activation including substantially heightened secretion of inflammatory mediators and phagocytic ability. These findings highlight the importance of investigating the influence of drug uptake on macrophage function and not making assumptions about the possible effects based on morphologic similarities to those drugs that have been associated with significant functional impairment and other adverse sequelae. Furthermore, based on the studies reported herein, it appears that the foamy (or vacuolated) appearance of AM following inhalation of AONs, as well as the influx of these cells within the alveolar space, may not be a deleterious process. Rather, it may be warranted to view this response as more of a normal, benign process of clearance of the inhaled material.

\section{References}

1. Nicklin PL, Bayley D, Giddings $\mathrm{J}$ et al. Pulmonary bioavailability of a phosphorothioate oligonucleotide (CGP 64128A): comparison with other delivery routes. Pharm Res 1998;15:583-91.

2. Templin M, Levin A, Graham $M$ et al. Pharmacokinetic and toxicity profile of a phosphorothioate oligonucleotide following inhalation delivery to lung in mice. Antisense Nucleic Acid Drug Dev 2000;10:359-68.

3. Duan W, Chan JH, McKay K et al. Inhaled p38alpha mitogen-activated protein kinase antisense oligonucleotide attenuates asthma in mice. Am J Respir Crit Care Med 2005;171:571-8.

4. Fortin M, Higgins $M$, Aubé $P$ et al. TPI 1100: an inhaled antisense oligonucleotide (AON) against Phosphodiesterase (PDE) 4 and 7 with significant antiinflammatory effects in a mouse model for COPD. Am J Respir Crit Care Med 2008; 177.

5. Nyce JW, Metzger WJ. DNA antisense therapy for asthma in an animal model. Nature 1997;385:721-5.

6. Gauvreau GM, Boulet LP, Cockcroft DW et al. Antisense Therapy against CCR3 and the Common Beta Chain Attenuates Allergen-induced Eosinophilic Responses. Am J Respir Crit Care Med 2008;177:952-8.

7. Monteith DK, Levin AA. Synthetic oligonucleotides: the development of antisense therapeutics. Toxicol Pathol 1999;27:8-13.

8. Henry SP, Grillone LR, Orr JL et al. Comparison of the toxicity profiles of ISIS 1082 and ISIS 2105, phosphorothioate oligonucleotides, following subacute intradermal administration in Sprague-Dawley rats. Toxicology 1997;116:77-88.

9. Guimond A, Viau E, Aube $P$ et al. Advantageous toxicity profile of inhaled antisense oligonucleotides following chronic dosing in non-human primates. Pulm Pharmacol Ther 2008;21:845-54.

10. Coonrod JD. Role of leukocytes in lung defenses. Respiration 1989;55 Suppl1:913.

11. Oberdorster G, Ferin J, Gelein R et al. Role of the alveolar macrophage in lung injury: studies with ultrafine particles. Environ Health Perspect 1992;97:193-9.

12. Nicolet-Chatelain G, Prevost MC, Escamilla R, Migueres J. Amiodaroneinduced pulmonary toxicity. Immunoallergologic tests and bronchoalveolar lavage phospholipid content. Chest 1991;99:363-9.

13. Hruban Z. Pulmonary changes induced by amphophilic drugs. Environ Health Perspect 1976;16:111-8. 
14. Reasor MJ, Kacew S. Drug-Induced Phospholipidosis: Are There Functional Consequences? Experimental Biology and Medicine 2001;226:825-30.

15. Myers JL, Kennedy JI, Plumb VJ. Amiodarone lung: pathologic findings in clinically toxic patients. Hum Pathol 1987;18:349-54.

16. Papiris SA, Triantafillidou C, Kolilekas L et al. Amiodarone: review of pulmonary effects and toxicity. Drug Saf 2010;33:53958.

17. Ott MC, Khoor A, Leventhal JP et al. Pulmonary toxicity in patients receiving low-dose amiodarone. Chest 2003;123:64651.

18. Bedrossian CW, Warren CJ, Ohar J, Bhan R. Amiodarone pulmonary toxicity: cytopathology, ultrastructure, and immunocytochemistry. Ann Diagn Pathol 1997;1:47-56.

19. Quinlan TR, BeruBe KA, Hacker MP et al. Mechanisms of asbestos-induced nitric oxide production by rat alveolar macrophages in inhalation and in vitro models. Free Radic Biol Med 1998;24:77888.

20. Chin BY, Trush MA, Choi AM, Risby TH. Transcriptional regulation of the $\mathrm{HO}-1$ gene in cultured macrophages exposed to model airborne particulate matter. Am J Physiol Lung Cell Mol Physiol 2003; 284:L473-80.

21. Schneider JC, Card GL, Pfau JC, Holian A. Air pollution particulate SRM 1648 causes oxidative stress in RAW 264.7 macrophages leading to production of prostaglandin E2, a potential Th2 mediator. Inhal Toxicol 2005;17:871-7.

22. Kim HJ, Lee HS, Chong YH, Kang JL. p38 Mitogen-activated protein kinase up-regulates LPS-induced NF-kappaB activation in the development of lung injury and RAW 264.7 macrophages. Toxicology 2006;225:36-47.
23. Vali H, Weiss B, Li YL et al. Formation of tabular single-domain magnetite induced by Geobacter metallireducens GS-15. Proc Natl Acad Sci U S A 2004;101:16121-6.

24. Halstead BW, Zwickl CM, Morgan RE et al. A clinical flow cytometric biomarker strategy: validation of peripheral leukocyte phospholipidosis using Nile red. J Appl Toxicol 2006;26:169-77.

25. Zamecnik PC, Stephenson ML. Inhibition of Rous sarcoma virus replication and cell transformation by a specific oligodeoxynucleotide. Proc Natl Acad Sci USA 1978;75:280-4.

26. Henry SP, Beattie G, Yeh G et al. Complement activation is responsible for acute toxicities in rhesus monkeys treated with a phosphorothioate oligodeoxynucleotide. Int Immunopharmacol 2002; 2:1657-66.

27. Henry SP, Novotny W, Leeds $\mathrm{J}$ et al. Inhibition of coagulation by a phosphorothioate oligonucleotide. Antisense Nucleic Acid Drug Dev 1997;7:503-10.

28. Jason TLH, Koropatnick J, Berg RW. Toxicology of antisense therapeutics. Toxicology and Applied Pharmacology 2004;201:66-83.

29. Levin AA. A review of the issues in the pharmacokinetics and toxicology of phosphorothioate antisense oligonucleotides. Biochim Biophys Acta 1999;1489:69-84.

30. Levin AA, Henry SP, Monteith DK, Templin M. Toxicity of antisense oligonucleotides. In: Crooke ST, ed. Antisense Drug Technology: Principles, Strategies, and Applications New York: Marcel Dekker, Inc.; 2001:201-67.

31. Gauldie J, Jordana M, Cox G. Cytokines and pulmonary fibrosis. Thorax 1993;48:931-5.

32. Wilson MS, Wynn TA. Pulmonary fibrosis: pathogenesis, etiology and regulation. Mucosal Immunol 2009;2:103-21.
33. Wynn TA. Cellular and molecular mechanisms of fibrosis. J Pathol 2008;214:199210.

34. Singh SN, Fletcher RD, Fisher SG et al. Amiodarone in patients with congestive heart failure and asymptomatic ventricular arrhythmia. Survival Trial of Antiarrhythmic Therapy in Congestive Heart Failure. N Engl J Med 1995;333:7782.

35. Abe A, Hiraoka M, Shayman JA. A role for lysosomal phospholipase A2 in drug induced phospholipidosis. Drug Metab Lett 2007;1:49-53.

36. Kasahara T, Tomita K, Murano $\mathrm{H}$ et al. Establishment of an in vitro high-throughput screening assay for detecting phospholipidosis-inducing potential. Toxicol Sci 2006;90:133-41.

37. Bargout R, Jankov A, Dincer E et al. Amiodarone induces apoptosis of human and rat alveolar epithelial cells in vitro. American Journal of Physiology - Lung Cellular and Molecular Physiology 2000; 278:L1039-44.

38. Tager AM, Kradin RL, LaCamera P et al. Inhibition of pulmonary fibrosis by the chemokine IP-10/CXCL10. Am J Respir Cell Mol Biol 2004;31:395-404.

39. Reasor MJ, McCloud CM, DiMatteo M et al. Effects of amiodarone-induced phospholipidosis on pulmonary host defense functions in rats. Proc Soc Exp Biol Med 1996; 211:346-52.

40. Hruban Z. Pulmonary and generalized lysosomal storage induced by amphiphilic drugs. Environ Health Perspect 1984;55: 53-76.

41. Gauvreau GM, Pageau R, Seguin R et al. Dose-response effects of TPI ASM8 in asthmatics after allergen. Allergy 2011;66: 1242-8. 